\title{
CANCELAMENTO DE CIRURGIAS PROGRAMADAS EM UM HOSPITAL-ESCOLA: UM ESTUDO EXPLORATÓRIO*
}

\author{
Jacqueline Borges Cavalcante** \\ Lorita Marlena Freitag Pagliuca*** \\ Paulo César Almeida****
}

CAVALCANTE, J.B.; PAGLIUCA, L.M.F.; ALMEIDA, P.C. Cancelamento de cirurgias programadas em um hospitalescola: um estudo exploratório. Rev.latino-am.enfermagem, Ribeirão Preto, v. 8, n. 4, p. 59-65, agosto 2000.

Apesar da vasta literatura disponível sobre o preparo do cliente cirúrgico e sobre a realização do ato cirúrgico, o enfoque dado à suspensão deste ato tem sido bastante restrito. Objetiva-se neste estudo identificar o quantitativo de cirurgias programadas e suspensas e os serviços mais afetados por esse acontecimento. O estudo foi realizado nos serviços de cirurgia de um hospital público de ensino, de grande porte, localizado na região metropolitana de Fortaleza, Ceará. Os dados foram levantados através dos livros de registro cirúrgico, mapas diários de programa cirúrgico e dos prontuários de clientes selecionados para serem submetidos à cirurgia no período de setembro a dezembro de 1996. Os dados levantados foram analisados quantitativamente e apresentados em tabelas. Os resultados demonstram que das 1.145 cirurgias programadas no periodo selecionado, 379 (33\%) foram suspensas. Os serviços mais prejudicados foram o de Cirurgia Geral, Oftalmologia, Cirurgia de Cabeça e Pescoço, Traumatologia e Ortopedia, Otorrinolaringologia, Nefrologia e Transplante Renal, e o de Proctologia. Faz-se necessário maiores investigações nessa área a fim de se conhecer as causas elou motivos que determinaram o cancelamento dessas cirurgias, bem como a participação da enfermagem no estudo dessa problemática.

UNITERMOS: enfermagem cirúrgica, pacientes, cuidados de enfermagem

\section{1- INTRODUÇÃO}

A realização de uma cirurgia é um acontecimento importante na vida de uma pessoa que encerra um significado próprio, já que através dela, essa pessoa espera viver de forma mais saudável, com melhor qualidade de vida.

Uma intervenção cirúrgica não é uma ocorrência rotineira na vida de um indivíduo. Ela requer um preparo prévio dessa pessoa e de seus familiares, que muitas vezes necessitam afastar-se de seus trabalhos, lares e de sua vida normal por algum tempo. Além disso, essas pessoas necessitam mobilizar recursos físicos, emocionais e até financeiros para enfrentar esse momento.

Qualquer cirurgia, mesmo aquela que é estética, feita por opção do indivíduo, cuja não realização não implicaria em prejuízos para sua saúde, tem importante significado e pode interferir com seu estado emocional.

Sob a ótica institucional, a programação de uma cirurgia envolve um número considerável de pessoas como cirurgiões, anestesiologistas, enfermeiros, auxiliares entre outros, além de grande quantidade de materiais e equipamentos especializados.

A suspensão de uma intervenção cirúrgica é uma ocorrência significante que nem sempre tem recebido a devida atenção por parte da equipe de saúde, bem como da administração do próprio hospital. Aparentemente, o cancelamento de cirurgias programadas não causa grande inquietação à equipe multiprofissional dos serviços de saúde, e os aspectos relativos à importância desse acontecimento para o paciente parecem estar esquecidos. A equipe encara esse acontecimento como rotineiro, inerente à estrutura organizacional e funcional da instituição.

Quando o indivíduo procura um hospital com um problema de saúde e lhe é indicada uma cirurgia como a solução para esse problema, ele aceita essa solução e marca sua cirurgia depositando na equipe de saúde e na instituição a certeza do apoio necessário para vencer mais essa etapa da sua vida. BRUSTCHER \& ZEN (1986, p.

\footnotetext{
* Estudo desenvolvido no Curso de Mestrado do DENF/UFC, apresentado no VI Colóquio Pan-Americano de Investigação em Enfermagem

** Enfermeira, Professor do DENF/UFC, mestranda

*** Enfermeira, Professor Titular do DENF/UFC

**** Estatístico, Professor Adjunto do DSC/UFC
} 
06) afirmam que "o paciente espera solução para os seus problemas por parte daqueles que teriam obrigação profissional e institucional de reconhecê-los e solucionálos".

O paciente cirúrgico ao ser hospitalizado traz consigo expectativas, dúvidas e temores sobre os acontecimentos que irá vivenciar. Ele estará em um ambiente estranho, com pessoas desconhecidas, longe de sua família e amigos. Seu corpo será agredido, cortado, mutilado. Muitas dúvidas e medos vêm à tona - Sentirei dor?, Ficarei curado?, Quanto tempo ficarei no hospital?, sendo que essas preocupações, em geral, estão voltadas para a realização da cirurgia e não para a suspensão da mesma. Por outro lado, o cancelamento de uma cirurgia programada implica em perda para a instituição com custo financeiro, atraso na programação cirúrgica e ainda prejuízo para outros pacientes que aguardam sua vez para operar.

Apesar da vasta literatura disponível abordando o preparo pré-operatório do paciente, assistência de enfermagem, fatores físicos e emocionais relacionados ao assunto, praticamente não há enfoque sobre a questão do cancelamento de cirurgias, surgindo a partir daí o interesse por essa temática.

É, portanto, nesse contexto que se delineiam os objetivos desse trabalho:

1.Levantar o número de cirurgias programadas e canceladas em um hospital escola no período de setembro a dezembro de 1996, e

2.Identificar, na instituição escolhida para o estudo, os serviços onde ocorrem cancelamentos de cirurgias.

\section{2- REVISÃO DE LITERATURA}

As finalidades dos procedimentos cirúrgicos são várias: de diagnóstico, de tratamento eletivo, de prevenção, de aliviar sintomas, de cura, de reconstrução ou cosmética. Advém que, qualquer ato cirúrgico por menor que seja sua extensão, desencadeia no indivíduo uma série de reações fisiológicas e psicológicas (BRUNNER \& SUDDARTH, 1993; BELAND \& PASSOS, 1978).

Uma cirurgia, mesmo quando consentida e programada significa uma agressão ao indivíduo, uma ameaça à sua integridade física e emocional. É notório o impacto de uma intervenção cirúrgica sobre o paciente, que na tentativa de extravasar seus sentimentos pode desenvolver quadros de angústia, depressão e isolamento.

Após a indicação da cirurgia, o paciente passa então a mobilizar todos os seus recursos físicos, emocionais e financeiros para enfrentar esse momento.
Muita energia é empregada nesse processo. A situação cirúrgica passa a ter agora um novo significado. Mesmo que a sua perspectiva seja angustiante e amedrontadora, ela passa a ser importante e emocionalmente necessária porque pode implicar em um estilo de vida mais saudável e com melhor qualidade de vida.

O estado emocional do paciente, a forma como ele se sente em relação à situação, influencia seu estado fisiológico podendo interferir com a cirurgia programada. Mason, citado por BRUTSCHER \& ZEN (1986), diz que a realização de uma cirurgia depende do trabalho de toda a equipe cirúrgica e que a enfermeira é o elemento que pode pressupor a emoção que o paciente pode sentir, apresentando um temor real que se estende até os membros da família. Como elemento chave dentro da estrutura hospitalar, a enfermeira tem condições de contribuir para que as cirurgias programadas sejam realizadas na data marcada e dentro de todas as condições de segurança requeridas.

Por outro lado, BELAND \& PASSOS (1978) lembram que nem sempre a enfermeira é capaz de satisfazer todas as expectativas do paciente e nem sempre é responsável por elas não serem atendidas. Ela é responsável por tentar identificá-las, levando-as em consideração no atendimento do mesmo.

Se a perspectiva de uma operação é um momento de crise e fator de ansiedade e estresse para o indivíduo, a frustração desse acontecimento é uma nova crise seguindo-se ou sobrepondo-se à primeira, que merece atenção especial da enfermeira. Em nossa experiência, a necessidade de uma cirurgia é percebida pelo paciente e familiares como uma situação extremamente desestabilizadora, que gera medo e ansiedade requerendo estratégias de adaptação, sendo que a não satisfação dessa necessidade pode gerar sentimentos de frustração que precisam ser trabalhados.

Outro aspecto importante da problemática da suspensão de cirurgias é abordado no estudo de ARIETA et al. (1995), quando afirmam que os recursos destinados à Saúde Pública no Brasil, além de serem insuficientes, são mal aproveitados. Os custos de manutenção e funcionamento dos hospitais públicos são cerca de $06 \mathrm{a}$ 10 vezes mais elevados que os hospitais privados e ainda assim funcionam com menor eficiência.

A suspensão de uma cirurgia implica em um custo operacional e financeiro para a instituição, tendo repercussões negativas no atendimento da população, principalmente daquela mais carente.

Evitar a suspensão de cirurgias através de uma assistência de enfermagem planejada e articulada com as demais equipes profissionais e da elaboração de um plano administrativo eficiente, deve ser um dos objetivos da assistência de enfermagem e da equipe administrativa da instituição hospitalar. Prestar assistência de 
enfermagem especial, para o cliente que teve sua cirurgia suspensa, deve se constituir em outra preocupação do profissional.

Constata-se pela revisão de literatura que o estudo do fenômeno suspensão de cirurgia contempla vários aspectos que, com certeza, não serão esgotados em uma única abordagem. Consideramos necessário desvelar o acontecimento para que se possa intervir na prevenção da suspensão do ato cirúrgico, ainda que se saiba que haverão circunstâncias tais em que as mesmas continuarão ocorrendo. Nosso objetivo final constitui-se em prestar cuidado de enfermagem centrado na Relação de Ajuda proposto por Ida Orlando, para a pessoa que teve sua cirurgia suspensa.

\section{3- METODOLOGIA}

Este estudo foi realizado em uma instituição pública federal que funciona como um hospital escola, localizado em Fortaleza-Ceará. Essa instituição foi escolhida porque, entre outras finalidades, opera como um núcleo de formação e aperfeiçoamento de profissionais ligados à área da saúde, atendendo não apenas à comunidade local mas, também, servindo como centro de referência para o interior do estado.

A coleta dos dados ocorreu no período de setembro a dezembro de 1996, junto aos livros de registro do Centro Cirúrgico e do SAME (Serviço de Arquivo Médico e Estatística), onde foram levantados o número de cirurgias programadas e suspensas, e identificadas as clínicas ou serviços onde ocorreram a maior incidência do cancelamento de cirurgias. Os dados foram dispostos em tabelas sendo então comentados. Para uma dessas tabelas fez-se o teste de X2.

\section{4- RESULTADOS E COMENTÁRIOS}

\section{1- Características da Instituição}

No momento do estudo, o hospital encontravase passando por grave crise financeira e com a unidade de emergência desativada. Esta instituição conta com 64 leitos cirúrgicos, sendo 28 leitos destinados à clientela masculina, 31 à clientela feminina e 5 leitos para pediatria, distribuídos em doze serviços: Cirurgia Geral, Oftalmologia, Cirurgia de Cabeça e Pescoço, Traumatologia e Ortopedia, Otorrinolaringologia, Nefrologia e Transplante Renal, Proctologia, Plástica, Pediatria e Neurologia.

A equipe de enfermagem das clínicas cirúrgicas é composta por 15 enfermeiras, 16 técnicos e 21 auxiliares de enfermagem. A equipe médica é composta por 37 cirurgiões e 21 residentes que, juntamente com 18 anestesiologistas, 3 enfermeiras, 13 técnicos de enfermagem, 15 auxiliares, 2 instrumentadoras de cirurgia, e 1 auxiliar de administração compõem a equipe do Centro Cirúrgico.

O Centro Cirúrgico possui 5 salas de operação que atendem a pacientes internados ou admitidos através do ambulatório de cirurgia. Este setor funciona das 7 às 17 horas, diariamente, de segunda a sexta-feira. À noite, nos finais de semana e feriados, atende somente os casos de emergência do próprio hospital.

O mapa cirúrgico diário é feito pela equipe de cirurgiões e entregue à secretaria do Departamento de Cirurgia que o envia à chefia de enfermagem do Centro Cirúrgico até às 14 horas do dia anterior. Cada serviço agenda suas cirurgias conforme a distribuição das salas de operação demonstrada no quadro a seguir:

\section{Quadro 1 - Programação das salas cirúrgicas por serviços, dia da semana e período do dia}

\begin{tabular}{|c|c|c|c|c|c|c|c|c|c|c|}
\hline SO & \multicolumn{2}{|c|}{ SEGUNDA } & \multicolumn{2}{|c|}{ TERÇA } & \multicolumn{2}{c|}{ QUARTA } & \multicolumn{2}{c|}{ QUINTA } & \multicolumn{3}{c|}{ SEXTA } \\
\hline & $\mathrm{M}$ & $\mathrm{T}$ & $\mathrm{M}$ & $\mathrm{T}$ & $\mathrm{M}$ & $\mathrm{T}$ & $\mathrm{M}$ & $\mathrm{T}$ & $\mathrm{M}$ & $\mathrm{T}$ \\
\hline 1 & OFT & OFT & OTOR & OFT & OTOR & OTOR & OTOR & OTOR & OFT & OTOR \\
\hline 2 & $\mathrm{CG}$ & $\mathrm{CP}$ & PROC & $\mathrm{CG}$ & PLAST & TO & PED & TO & URO & CG \\
\hline 3 & TO & CG & TX & TO & CP & CP & CG & CG & TO & TO \\
\hline 4 & CG & CG & TX & URO & PROC & CG & URO & CG & CP & CP \\
\hline 5 & CV & PLAST & CV & PLAST & CG & CG & PROG & PROG & VAS & VASC \\
\hline
\end{tabular}

Obs: SO (Sala de Operação), OFT (Oftalmologia), OTOR (Otorrinolaringologia), CG (Cirurgia Geral), CP (Cabeça e Pescoço), PROC (Proctologia), PLAST (Plástica), TO (Traumatologia e Ortopedia), PED (Pediatria), URO (Urologia), TX (Transplante Renal e Nefrologia), CV (Cardio-Vascular), VASC (Vascular) 


\subsection{Características dos serviços de cirurgia}

O serviço de Cirurgia Geral atende a uma grande demanda de pacientes internos e externos, de ambos os sexos, realizando vários tipos de cirurgia, inclusive as ginecológicas. A clientela atendida nesse serviço é, com freqüência, portadora de patologias graves e debilitantes, requerendo exames mais específicos e demorados, necessitando de assistência médica e de enfermagem mais intensivas. Por vezes esses pacientes permanecem por um longo período de pré-operatório até que estejam em condições de enfrentar a cirurgia.

Nesse setor, a maior parte das cirurgias programadas é de grande porte, sendo que ocorrem também cirurgias de médio e pequeno portes. Estas últimas, apesar de necessitarem de menor preparo do paciente, envolvem um grande número de pessoas, equipamentos e salas de operação.

O serviço de Oftalmologia também atende a uma grande demanda de pacientes, adultos e crianças, de ambos os sexos. Nesse serviço, embora ocorram cirurgias de grande porte, predominam as cirurgias de médio porte, ocorrendo ainda cirurgias de pequeno porte e exames sob sedação, que não necessitam da internação do paciente. Quando são operados no ambulatório, esses pacientes, em geral, permanecem pouco tempo no hospital, recebendo alta logo após a realização da cirurgia. Contudo, como a rotatividade desses pacientes é muito elevada, e podem ser marcadas várias cirurgias por dia, há necessidade de um maior número tanto de profissionais quanto de equipamentos e de salas de operação disponíveis.

O serviço de Cirurgia de Cabeça e Pescoço atende adultos e crianças de ambos os sexos. Os pacientes são em geral portadores de patologias graves e debilitantes, necessitando internação hospitalar mais demorada tanto no período pré quanto no período pós-operatório, o que faz com que sejam marcadas poucas cirurgias por dia e seus leitos permaneçam ocupados por períodos mais longos.

O serviço de Traumatologia e Ortopedia, assim como os serviços anteriores, atende a pacientes de ambos os sexos, inclusive crianças. As cirurgias aqui programadas são principalmente de grande e médio portes. Nesse serviço, todos os pacientes necessitam de internação hospitalar, e muitos passam por longos períodos de internação, para que possam ser melhor acompanhados durante sua convalescença.

No serviço de Otorrinolaringologia são atendidos pacientes adultos e crianças, de ambos os sexos. Esses pacientes, em geral, encontram-se em boas condições clínicas. Contudo, alguns deles, quando as cirurgias programadas são de grande porte, necessitam de internação hospitalar. É o caso dos pacientes portadores de infecção da mastóide, que necessitam de um maior tempo de tratamento antes de se submeterem à cirurgia. Nesse serviço podem ser programadas várias cirurgias por dia.

A clínica de Nefrologia funciona juntamente com o serviço de Transplante Renal. Nesses setores é atendido um grande número de pacientes, tanto adultos quanto crianças, de ambos os sexos. Esses pacientes caracterizam-se por serem portadores de patologias graves e crônicas. Muitos deles possuem outras doenças associadas à doença de base, o que faz com que necessitem de assistência médica e de enfermagem rigorosas e longos períodos de internação. Também é atendido aqui um grande número de pacientes externos, provenientes de outros hospitais, que são encaminhados a esse serviço para se submeterem à confecção de fístula artério-venosa (FAV). Esse procedimento, necessário para a realização da hemodiálise, apesar de simples e rápido, deve ser feito no Centro Cirúrgico.

As cirurgias realizadas no serviço de Nefrologia são muito variadas, abrangendo aquelas de grande a pequeno porte, como as biópsias. Os transplantes renais são cirurgias extensas, demoradas, que envolvem grande número de profissionais e equipamentos, e ocupam simultaneamente duas salas de operação. Essas cirurgias, uma vez programadas, raramente são canceladas, já que só são aprazadas quando os pacientes encontram-se em condições favoráveis de serem operados, e quando a equipe se científica de que todos os requisitos necessários para a realização da cirurgia foram atendidos.

A Proctologia é um serviço com clientela predominantemente adulta, dos sexos masculino e feminino. Os pacientes atendidos encontram-se, em geral, em boas condições clínicas não necessitando de um longo período de internação antes de se submeterem à cirurgia. As cirurgias programadas nesse serviço são contaminadas, e principalmente de médio e pequeno portes, mais simples e rápidas, podendo ser realizadas várias cirurgias por dia, sendo que após o término de cada cirurgia deve ser feita a limpeza e desinfecção da sala de operação.

\section{3- Quantitativo de cirurgias suspensas}

Tabela 1- Número de cirurgias programadas e suspensas em um hospital escola no período de setembro a dezembro de1996, Fortaleza-Ce

\begin{tabular}{lccc}
\hline MÊS & PROGRAMADAS & $\begin{array}{c}\text { CIRURGIAS } \\
\text { SUSPENSAS }\end{array}$ & S/P(\%) \\
\hline SET. & 356 & 97 & 27 \\
OUT. & 299 & 86 & 29 \\
NOV. & 279 & 111 & 40 \\
DEZ. & 211 & 85 & 40 \\
TOTAL & 1145 & 379 & 33 \\
\hline
\end{tabular}


Pela Tabela 1 pode-se verificar que de setembro a dezembro de 1996 foram programadas um total de 1145 cirurgias, sendo que destas 379 (33\%) foram suspensas, constituindo um número bastante elevado de suspensões. Em termos percentuais, o número de cirurgias canceladas foi crescente $(27,29,40$ e $40 \%)$ nos meses pesquisados. Verifica-se ainda que, nos meses de novembro e dezembro, houve uma tendência maior no número de suspensões, isto é, em novembro e dezembro as porcentagens são significativamente mais altas $(\mathrm{x} 2=9,3$; $\mathrm{p}=0,03)$.

Uma explicação para esse crescimento progressivo no número de cirurgias canceladas pode ser justificado devido a grave crise financeira pela qual passava a instituição. Outra explicação encontrada é que no final do ano, principalmente no mês de dezembro, com a chegada dos feriados natalinos e com os festejos e eventos relacionados aos mesmos, diminui o número de cirurgias programadas (211 em dezembro contra 356, 299 e 279 em setembro, outubro e novembro, respectivamente), aumentando o número relativo de cirurgias suspensas $(40 \%$ em novembro e dezembro contra $27 \%$ e $29 \%$ em setembro e outubro). Nesse período, muitos profissionais aproveitam para tirar suas férias e os pacientes optam por adiar cirurgias não urgentes para poder passar os feriados natalinos com seus familiares.

Devido a grande demanda de clientes neste hospital, muitos pacientes têm suas consultas, internamentos e cirurgias aprazados, às vezes, com meses de antecedência. Quando esse calendário é estabelecido, os acontecimentos anteriormente referidos, eventos festivos por exemplo (com excessão dos feriados nacionais), não são considerados, provocando transtornos no planejamento, assim como os exames pré-operatórios não têm mais validade.

Nos tempos atuais, quando a maioria das instituições e empresas tem se voltado para a melhoria na qualidade dos serviços oferecidos à população, não se admite numa instituição prestadora de serviços de saúde o planejamento não seja etapa obrigatória do seu funcionamento operacional.

Pela Tabela 2 pode-se comparar o número de cirurgias programadas e suspensas nos vários serviços do hospital durante os meses de setembro a dezembro de 1996.

Tabela 2 - Número de cirurgias programadas e suspensas por serviço/clínica em um hospital escola no período de setembro a dezembro de 1996, Fortaleza-Ceará

\begin{tabular}{|c|c|c|c|c|c|c|c|c|c|c|c|c|}
\hline \multirow{2}{*}{$\underset{f}{\mathrm{SERV}}$} & \multicolumn{12}{|c|}{ MÊS } \\
\hline & \multicolumn{3}{|c|}{ SET } & \multicolumn{3}{|c|}{ OUT } & \multicolumn{3}{|c|}{ NOV } & \multicolumn{3}{|c|}{$\mathrm{DEZ}$} \\
\hline $\mathrm{CG}$ & 100 & 18 & 18 & 96 & 20 & 20 & 88 & 39 & 44 & 65 & 28 & 43 \\
\hline OFT & 37 & 12 & 32 & 24 & 16 & 47 & 27 & 14 & 52 & 30 & 11 & 37 \\
\hline TO & 38 & 15 & 39 & 27 & 12 & 44 & 25 & 07 & 28 & 11 & 04 & 36 \\
\hline OTOR & 38 & 08 & 21 & 29 & 07 & 24 & 28 & 11 & 24 & 25 & 06 & 24 \\
\hline $\mathrm{NEF} / \mathrm{TX}$ & 29 & 07 & 24 & 20 & 06 & 30 & 24 & 06 & 25 & 16 & 13 & 81 \\
\hline PROC & 27 & 12 & 44 & 16 & 04 & 25 & 20 & 05 & 25 & 17 & 08 & 47 \\
\hline TOTAL & 354 & 97 & 27 & 299 & 86 & 29 & 278 & 111 & 39 & 210 & 85 & 40 \\
\hline
\end{tabular}

$\mathrm{X}^{2}=90,11 ; \mathrm{P}=0,000$

a) CG - Cirurgia Geral; OFT - Oftalmologia; CP - Serviço de Cirurgia de Cabeça e Pescoço; TO - Traumatologia e Ortopedia; OTOR - Otorrinolaringologia; NEF/TX - Nefrologia e Transplante Renal; PROC - Proctologia

b) Cardio-Vascular; Plástica; Pediatria; Urologia

Os serviços que mais se destacam mantendo elevados percentuais de suspensão em todos os meses pesquisados são: o de Cirurgia Geral (18, 20, 44 e 43\%), o de Oftalmologia (32, 47, 54 e 37\%), o de Cirurgia de Cabeça e Pescoço $(42,59,50$ e 50\%) e o de Traumatologia e Ortopedia (39, 44, 28 e 36\%). Contudo, mesmo em menor escala, os serviços de Otorrinolaringologia, Nefrologia e Transplante Renal e Proctologia também foram afetados pela suspensão de cirurgias. No item OUTROS foram agrupados os serviços de cirurgia Cárdio-Vascular, Plástica, Pediatria e Urologia, por apresentarem isoladamente baixos índices de suspensão. Finalizamos esses comentários ressaltando que todos os serviços pesquisados foram atingidos pelo cancelamento de cirurgias, causando prejuízos que afetam não apenas a instituição, mas principalmente a clientela que atende.

Na era da "Qualidade Total" é inadmissível que centenas de pessoas deixem de ter suas necessidades de saúde assistidas, e ainda que tantos recursos financeiros sejam perdidos ou mal aproveitados em um região que tem como um dos seus principais problemas a baixa 
qualidade da atenção à saúde da população.

Como provedores de cuidados de saúde, cabe a cada enfermeiro a responsabilidade de participar do planejamento da instituição contribuindo para que esta forneça à clientela os serviços necessários, além de oferecer assistência de enfermagem especializada.

As instituições de saúde possuem objetivos e responsabilidades para com o indivíduo em particular, sua família e a comunidade, e é no cumprimento desse objetivos e responsabilidades que têm sua existência e manutenção justificadas.

O controle adequado das cirurgias agendadas proporcionará menor espoliação do paciente, diminuição no tempo de internação e dos riscos de infecção hospitalar, redução dos custos do tratamento com benefícios diretos para os usuários que serão melhor assistidos, e para a instituição que terá sua produtividade e retorno financeiros aumentados.

\section{5- CONCLUSÕES}

Esse estudo permitiu identificar, na instituição pesquisada, que das 1145 cirurgias programadas no período do estudo, 379 (33\%) foram suspensas. Os serviços que mais se destacaram mantendo elevados percentuais de suspensão em todos os meses pesquisados foram o de Cirurgia Geral $(18,20,44$ e $43 \%)$, o de Oftalmologia (32, 47, 54 e 37\%), o de Cirurgia de Cabeça e Pescoço $(42,59,50$ e $50 \%)$ e o de Traumatologia e Ortopedia $(39,44,28$ e 36\%).

Faz-se necessário maiores investigações nessa área a fim de se conhecer as causas e/ou motivos que determinaram o cancelamento dessas cirurgias, bem como a intensificação da participação da enfermagem no estudo dessa problemática.

Tem-se que repensar o papel das grandes instituições de saúde no atendimento ao paciente cirúrgico, motivando debates entre usuários e profissionais. A enfermagem precisa refletir sobre essa problemática, contribuindo com novas informações e sugestões, e atuando de forma sincronizada com outros profissionais, num trabalho de equipe, tendo um objetivo comum: oferecer ao paciente o cuidado que ele merece.

\section{CANCELLATION OF SCHEDULED SURGERIES IN A UNIVERSITY HOSPITAL: AN EXPLORATORY STUDY}

In spite of the extensive available literature on surgery patients' preparation and on the performance of surgeries, the focus given to the cancellation of the surgical act has been quite restricted. This study aims at identifying the number of scheduled and cancelled surgeries as well as the services that are mostly affected by such cancellations and was carried out in the surgery service of a big public university hospital located in the metropolitan area of Fortaleza, Ceará. The data were collected through surgery registration books, daily maps of surgery schedules and from the files of patients scheduled for surgery from September to December, 1996. The gathered data were analyzed quantitatively and introduced in charts. The results demonstrate that from the 1,145 surgeries programmed in the selected period, 379 (33\%) had been cancelled. The mostly prejudiced services were General Surgery, Ophthalmology, Head and Neck Surgery, Trauma and Orthopedics, Otorhinolaryngology, Nephrology and Renal Transplant, and Proctology. Further investigation in this area in order to know the determinant causes of surgery cancellation as well as the participation of nursing in the study of this problem are necessary.

KEY WORDS: surgical nursing, patients, nursing care

\section{LA CANCELACIÓN DE CIRUGÍAS PROGRAMADAS EN UN HOSPITAL-ESCUELA: UN ESTUDIO EXPLORATORIO}

A pesar de la literatura disponible traer un inmenso abordaje en relación con la preparación del cliente para el acto quirúrgico, el enfoque dado a la suspensión de este acto se ha restringido.. Se busca identificar la cantidad de cirugía fijadas y suspendidas y los servicios más afectados por ese evento. El estudio se realizó en los servicios de cirugía de un hospital público de enseñanza, de grande porte, localizados en el área metropolitana de Fortaleza, Ceará. Los datos fueron recogidos a través de los libros de registro quirúrgico, mapas diarios de programa quirúrgico y de las historias clínicas de los clientes seleccionados y sometidos a la cirugía en el periodo de septiembre a diciembre de 1996. Se analizaron los datos cuantitativamente y se presentaron en tablas. Los resultados demuestran que de las 1.145 cirugías programadas en el periodo seleccionado, 379 (33\%) fueron suspendidas. Los servicios afectados fueron: Cirugía General, Oftalmología, Cirugía de Cabeza y Cuello, Traumatología y Ortopedia, Otorrinolaringología, Nefrología y Trasplante Renal, y Proctología. Se hacen necesarias investigaciones en esta área para saber las razones que determinaron la cancelación de esas cirugías, así como la participación de enfermería en el estudio de esta problemática. 
6- REFERÊNCIAS BIBLIOGRÁFICAS

01. ARIETA, C.E.L.; TAIAR, A.; KARA JOSÉ, N. Utilização e causas de suspensão de intervenções cirúrgicas oculares em centro cirúrgico ambulatorial universitário. Rev. Assoc. Med. Bras., v. 41, n. 3, p. 233-5, 1995.

02. BELAND, I.; PASSOS, J. Enfermagem clínica. São Paulo: EPU/EDUSP, 1978. v. 1.
03. BRUNNER, L.S.; SUDDARTH, D.S. Tratado de enfermagem médico cirúrgica. 7 . ed. Rio de Janeiro: Guanabara Koogan, 1993. v. 1, p. 34358.

04. BRUSTCHER, S.M.; ZEN, O.P. Humanização: enfermeira de centro cirúrgico e o paciente de cirurgia. Enfoque, v. 14, n. 1, p. 4- 6, set. 1986. 